\title{
Implications of Pollinator Biodiversity Decline for Food Security, Economy, and Pollinator Conservation Policies
}

\author{
Denis Vasiliev ${ }^{*}$ \\ 1Turiba University, 68 Graudu street, LV-1058, Riga, Latvia
}

\begin{abstract}
Pollinator decline is one of the most significant ecological problems of the $21^{\text {st }}$ century. This decline threatens human food security and global economy. In order to address this problem governments across Europe and the USA have introduced national pollinator conservation strategies. These strategies, however, significantly differ in approaches to conservation. The differences at least in part stem from lack of consensus in the literature on whether pollinator biodiversity or abundance of a few common species determines crop pollination. Critical evaluation of empirical evidence available to date outlined in this paper suggests that pollinator biodiversity rather than abundance of dominant species determine quality, magnitude and resilience of pollination ecosystem services to agriculture. In order to maintain pollinator biodiversity conservation strategies and initiatives should focus on enhancing habitat quality, complementarity and connectivity, rather than solely on increasing floral resource abundance and diversity within farmland fields. Conservation strategies currently underway need to be improved to address all three factors through landscape scale interventions. Countries that intend to design and introduce pollinator conservation strategies should take best practices from several existing strategies rather than choosing a single strategy as an example.
\end{abstract}

\section{Introduction}

Contribution of pollination ecosystem service to global economy exceeds 1.5 billion USD, which is equivalent to about $10 \%$ of the value of the world agricultural output [1]. Numerous studies found that pollination by wild bees Hymenoptera, flies Diptera, and butterflies and moths Lepidoptera, is more significant than by domestic pollinators $[2,3,4]$. At the same time, recent global pollinator assessment carried out by the International Science-Policy Platform on Biodiversity and Ecosystem Services revealed that pollinator biodiversity is in decline all over the world [5]. This situation is alarming, and it has stimulated governments of numerous countries and regions to design and start implementing national and regional pollinator conservation strategies and initiatives [6]. However, approaches to pollinator conservation significantly differ in their capacity to protect pollinator diversity [7]. These differences at least in part may be a result of lack of consensus in the literature on whether pollinator biodiversity or abundance of common species ensures pollination ecosystem service [8], as well as differences in opinion on whether landscape pattern or solely floral resource availability is important for maintaining pollinator biodiversity [9]. Lack of consistency in conservation approaches is likely to result in uncoordinated activities that may fundamentally undermine effectiveness of pollinator biodiversity conservation at larger spatial scales [10]. Understanding the role of pollinator biodiversity in delivery of pollination ecosystem service to agriculture and resulting contribution to human food security is essential for effective conservation intervention design [11]. Discords, in the literature may limit effectiveness of pollinator conservation due to differences in local approaches undermining implementation of coordinated conservation efforts at larger scales (i.e. regional and transnational). Moreover, lack of clarity may accommodate vested interests of stakeholder groups opposing complex conservation interventions essential for pollinator biodiversity conservation. Thus, the aim of this paper is to discuss the ways in which pollinator biodiversity contributes to pollination ecosystem service and to evaluate effectiveness of pollinator conservation strategies currently underway in terms of their capability to maintain pollinator diversity. For this purpose, first empirical evidence on contribution of pollinator biodiversity to crop pollination is discussed. After that factors important for maintaining pollinator diversity at a landscape scale are discussed. Finally, pollinator conservation strategies are critically evaluated.

\section{Pollinator species diversity and pollination ecosystem service}

A widespread opinion suggests that abundance of few dominant pollinator species, rather than pollinator species diversity, ensures crop pollination [12]. However, recent empirical evidence indicates that species diversity

\footnotetext{
* Corresponding author: denisksil@gmail.com
} 
determines crop pollination quality, magnitude, and resilience. Numerous studies found niche complementarity to be the mechanism that determines pollination quality, yield stability and productivity of agricultural crops [e.g. 13,14,15]. This phenomenon was observed in strawberry, apple, cherry, oilseed rape, bean, and sunflower, suggesting that it is most likely common to pollinator depended crops.

Nevertheless, some authors argue that the magnitude of pollination ecosystem service is determined by abundance of few dominant species [16]. However, recent findings by Winfree et al [17] suggest that dominant species determine sufficient level of pollination at a single field scale, while at a landscape scale a full regional species pool is essential for sufficient pollination magnitude. These findings indicate that the number of species essential for effective crop pollination increases with spatial scale.

Another important aspect of pollination is its resilience to a global environmental change. In line with Biodiversity Insurance Hypothesis [18] biodiversity sustains delivery of ecosystem services under stress. However, is this the case for pollination service to agriculture? Indeed, substantial amount of empirical evidence proves relevance of the hypothesis to crop pollination. For instance, a number of studies $[19,20,21]$ have found that response diversity in species rich pollinator assemblages buffers crop pollination against extreme weather events. Moreover, several authors [22, 23] revealed that inter-annual complementarity of pollinator species phenology ensures resilience of pollination delivery in the context of climate change driven shifts in plant phenology in the Northern Hemisphere. Furthermore, Winfree and Kremen [24] have found that due to differences in pollinator species dispersal capabilities may buffer pollination service from negative impact of landscape fragmentation. Overall, the evidence discussed above suggests that pollinator biodiversity critical for determining pollination service quality, magnitude, and resilience. Thus, the diversity rather than abundance of common pollinator species underpins human food security. It is, therefore, important to understand which factors maintain pollinator biodiversity at landscape and regional scales.

\section{Causes of pollinator biodiversity loss}

Scientific understanding of insect pollinator decline causes is still incomplete [25]. A broad array of factors is frequently cited as likely direct drivers of pollinator decay. However, there seems to be no consensus in the literature about relative importance of these stressors [26]. Furthermore, recent findings indicate that different drivers of pollinator decline act in synergy, making addressing impacts of different factors even more complex [27]. Nevertheless, numerous authors refer to landscape alteration [e.g. 28] and excessive pesticide application [e.g. 29] as to major immediate drivers of pollinator decay. This assumption is supported by the fact that the most intensively farmed geographical areas coincide with the sharpest pollinator decline areas [30].
Land management and pesticide use tend to be directly targeted by pollinator conservation policies [31]. However, while there seems to be relative consensus on harm of pesticides, significant disagreements surround impacts of different aspects of land use change [24]. In addition, it is important to note that landscape homogenization often leads to loss of natural enemies of crop pests and increased need to apply pesticides. Therefore, landscape alteration merits particular attention.

Pollinator decline may be driven by loss, fragmentation or degradation of natural habitats [32]. Studies on natural habitat loss demonstrate that decline of foraging and nesting resources have negative impacts on pollinator communities [33]. Still, loss of natural floral resources may be mitigated to some extent, as diversified agricultural landscapes were found to support speciesrich pollinator communities [34]. At the same time, hypothesis exists that loss of nesting resources may be equally or even more detrimental to pollinators than shortage of forage [35]. For instance, about three quarters of bee species are ground nesting and tillage may destroy their nests [36].

Despite the fact, that natural and semi-natural landscapes play a central role in sustaining wild pollinator biodiversity, surprisingly few studies consider impacts of land use changes within such landscapes [5]. Forest patches are often the main type of natural habitat in temperate ecosystems, and provide multiple insect pollinator species with nesting and reproduction sites [37]. Furthermore, pollinator species nesting in open landscapes depend on forests for over-wintering sites, refugia during adverse weather conditions and permanent foraging resources [38]. It is important to remember, however, that not only deforestation, but also forest growth may drive to pollinator decline. Several studies have revealed that due to lack of natural disturbances (e.g. wildfire) structural changes in temperate forest canopies may cause decline in pollinator species richness $[39,40]$. Furthermore, widespread forest re-growth on abandoned agricultural lands leads to loss of natural meadows and High Natural Value farmlands (HNVfs), which tend to harbor diverse pollinator communities [41]. Ignorance of afforestation risks for pollinators may be particularly detrimental in the context widespread climate mitigation strategies, where governments provide subsidies for forest plantation establishment on abandoned lands.

Relatively large proportion of land has been converted for urban infrastructure in Europe and in the U.S. [42]. Urban areas are expanding and this land use change may contribute to wild pollinator decline [43]. Nevertheless, some studies demonstrate that heterogeneous urban landscapes with substantial proportion of green spaces can support biodiverse pollinator assemblages [e.g. 44]. Bee species richness in urban and sub-urban landscapes can even exceed that in agricultural lands [45]. Thus, it appears most plausible that any anthropogenic land use change can decrease pollinator biodiversity in case such change leads to landscape homogenization and associated loss of diverse nesting and forage resources.

Global climate change is expected to increase risk of multiple pollinator species extinctions in the nearest future [46] and most likely already plays a significant 
role in pollinator decline [47]. However, climate adaptation activities are often omitted in pollinator conservation strategies. In part this ignorance may be explained by scarcity of studies proving impacts of climate change on pollinators and pollination. Still, it is important to remember that insect pollinators are ectoterms and tend to be sensitive to changes in ambient temperatures. Several transcontinental studies already report range counteractions of bumblebees [48] and butterflies [49] across Northern hemisphere. This happens due to range losses at the trailing latitudal range limits and insufficient expansion at northern range limits [5]. Bioclimatic envelope models for butterflies [50] and bumblebees [51] indicate that even under most optimistic climate change scenarios pollinator species will need to migrate substantial distances in order to keep pace with species thermal limits. Inability to tackle climate change is likely to result in multiple species extinctions [50]. Furthermore, empirical evidence proves that extreme weather events, including droughts [52] and heat waves [53], negatively affect pollinators or can limit their resource base. Landscape fragmentation and loss of diverse habitats with different microclimates are likely to reduce ability of pollinators to escape adverse impacts of climate change [54]. Thus, there seems to be a consensus between the authors that impacts of climate change are likely to be particularly damaging in synergy with the land use change and other drivers of pollinator decline [5].

As follows from the facts discussed above, land use change can simultaneously tackle multiple other drivers of pollinator decline; therefore, landscape level interventions appear to be pivotal to pollinator conservation success. However, approaches to pollinator conservation differ in their capability maintain pollinator species diversity [55]. Concerns exist that widespread measures applied for sustaining pollination services benefit few common species [56].

\section{Factors important for maintaining pollinator biodiversity}

Many empirical studies demonstrate that landscape composition can determine pollinator diversity [e.g. 57, 58]. However, a recent meta-analysis [59] has found that only drastic habitat losses have negative impacts on bee abundance and species richness. Referring to this metaanalysis, Roulston and Goodell [60] argue that the most important limiting factor affecting bee population abundance and species richness is availability of food resources. If this hypothesis holds true, conclusion can be made that increasing amount and diversity of floral resources within agricultural fields could be the main tool of pollinator conservation [61]. However, bees, as well as other pollinator taxa, are multi-habitat species, requiring different resources for nesting/reproduction and foraging during their lifecycle [62]. Furthermore, pollinator communities consist of multiple species with different habitat requirements (e.g. cavity nesting bees and saproxilic hoverflies vs. ground nesting bees), and also range greatly in species mobility within each taxonomic group. Therefore, it seems plausible that maintaining habitat heterogeneity, rather than simply increasing amount of floral resources within agricultural fields, is essential for conservation of pollinator biodiversity. Landscape complexity appears to be particularly important in sustaining species rich pollinator communities in temperate ecosystems, where pollinator species tend to be adapted to landscape fragmentation [63]. Indeed, in concordance with a plausible hypothesis articulated by Pykala [64] historically biodiversity in temperate realm was maintained by heterogeneous landscape mosaics. Complex landscape patterns in prehistoric times were shaped by large scale natural disturbances (e.g. fires and large herbivores). After suppression of natural disturbances by humans up until current expansion of intensive agriculture landscape heterogeneity was maintained by traditional extensive farming, thereby supporting biodiversity.

Still, given the fact that landscape heterogeneity is often shaped by disturbances, different opinions exist on how landscape alteration may impact pollinator biodiversity [65]. These concerns, in fact, are rooted in fundamental scientific debate on whether ecosystems should be managed towards equilibrium or dynamic state in order to support species diversity [66]. Empirical evidence, tends to support Connell's Intermediate Disturbance Hypothesis [67], which suggests that landscape heterogeneity shaped by moderate disturbances can maintain the highest species richness [68]. For instance, numerous studies in temperate forests $[69,70]$ and rangelands $[71,72]$ have revealed that succession and resulting landscape homogenization lead to pollinator diversity decline. At the same time, moderate disturbances such as grazing [73], fire [74], forest thinning and selective logging [75], if resulting in heterogeneous landscape mosaic, increase pollinator community richness.

Increasing habitat complexity is often associated with increase in floral resource availability, making it hard to disentangle positive impacts of these changes on pollinator communities. Nevertheless, a number of facts prove that structural landscape heterogeneity per se plays a significant role in supporting pollinator biodiversity. First of all, Chesson's coexistence theory [76], suggests that heterogeneous ecosystems are able to support greater species richness than homogenous due to reduced competition between species at the same trophic level. Indeed, both mathematical models [77] and empirical evidence [78] proves that landscape heterogeneity facilitates niche partitioning in pollinator communities. Secondly, complex landscapes usually provide pollinators with multiple refugia, reducing risks of e.g. predation or exposure to pesticides [79]. Thirdly, heterogeneous landscapes usually provide wide range of microclimatic conditions [80]. Recent findings indicate that structural heterogeneity of habitat types and topography may buffer pollinator communities from negative impacts global warming and extreme weather events [81].

Contribution of landscape heterogeneity to pollinator species richness and abundance becomes even more evident when considering interplay between different ecosystem types at multiple spatial scales. Fahrig et al. [82] suggest that while species richness at a local scale 
can be maximized trough heterogeneity within the landscape, biodiversity at a regional scale depends on diversity of very large patches, requiring presence of both structurally homogenous and heterogeneous landscapes. Empirical evidence indicates validity of this hypothesis for pollinator communities. For instance, several metaanalyses [83, 84] demonstrate that diversified farming systems contribute positively to pollinator biodiversity when surrounded by homogenous, rather than heterogeneous landscapes. Similarly, "ecological contrast" was found to support species rich pollinator communities in relatively homogenous crop fields [85] and fruit orchards [86] located in vicinity of heterogeneous natural or semi-natural landscapes. As follows from the facts discussed above landscape context rather than solely diversity and abundance of floral resources is important for maintaining pollinator biodiversity. Thus, it is important to understand whether pollinator conservation strategies currently underway are capable of maintaining pollinator biodiversity.

\section{Conservation strategies}

Multiple pollinator conservation strategies are currently implemented across Europe and the USA [88]. Most of these strategies place a particular emphasis on a need for increasing amount and abundance of floral resources within agricultural landscapes, but often ignore landscape pattern itself [88]. However, according to a comprehensive study by Cole et al [87] habitat quality, connectivity and complementarity maintain pollinator biodiversity within landscapes in temperate ecosystems. Habitat quality for pollinators may be enhanced through increasing availability of abundant and diverse floral resources, although preservation of natural and seminatural habitats may be more effective than conservation interventions based on flower plantations proposed in most pollinator conservation strategies [55].

Habitat complementarity is considered in many national policies emphasizing the need to preserve pollinator habitats across different land-uses; however, forest habitats are omitted in all but Norwegian, German, French, the US, and Scottish strategies. This is particularly worrying, as forests often harbor biodiverse pollinator assemblages, which spill-over to agricultural fields [89, 90]. Moreover, pollinator species nesting in open landscapes depend on forests for over-wintering sites, refugia during adverse weather conditions and permanent foraging resources [91]. Across North Temperate Zone more than a half of land area has historically been converted to agriculture, with small areas of forest remnants persisting within less intensively managed agricultural landscapes [92]. Natural disturbances within large remaining forest areas (e.g. wildfire) were suppressed long ago [93]. Although, forest loss and fragmentation continues, it has slowed down in recent decades, due to establishment of forest plantations and forest re-growth on abandoned lands [94, 95]. It is therefore important to remember that both forest loss and forest re-growth may drive pollinator decline [96, 97]. Several studies have revealed that due to lack of natural disturbances structural changes in temperate forest canopies may limit proportion of open spaces and cause decline in pollinator species richness [98, 99]. Also, forest re-growth on abandoned agricultural lands leads to loss of natural meadows and High Nature value farmlands, which tend to harbor diverse pollinator communities [100]. Ignorance of afforestation risks for pollinators may be particularly detrimental in Eastern Europe, where abandoned lands are widespread and in the context of climate mitigation strategies EU subsidies may be used for forest plantation establishment on such lands [101].

Landscape connectivity, perhaps, is the area of particular discrepancy between national pollinator conservation strategies. Many strategies simply omit this factor. In the UK strategies, however, importance of landscape connectivity is emphasized with "B-Lines" initiative implemented across England, Scotland and Wales is specifically designed to ensure landscape connectivity for pollinators. German and French strategies also include recommendations to preserve habitat networks for pollinators, which indeed could result in improved landscape connectivity. German strategy also suggests preservation of eco-tones between habitat types, the factor that may be particularly important for improving landscape connectivity pollinators. The pollinator strategy for Scotland emphasizes the need for multi-scale approach. Overall, it appears that none of the strategies currently underway represents a perfect model for other countries, rather several strategies together may serve as an example for other countries that intend to design and implement pollinator conservation at a national scale.

\section{Conclusions}

The aim of this study was to discuss the ways in which pollinator biodiversity contributes to pollination ecosystem service and to evaluate effectiveness of pollinator conservation strategies currently underway. Review of currently available empirical evidence suggests that pollinator biodiversity rather than abundance of common species most likely determines quality, magnitude, and resilience of crop pollination. This fact provides justification for complicated pollinator conservation interventions, as preserving pollinator biodiversity will ensure human food security and deliver economic benefit. Most national pollinator conservation strategies currently implemented across Europe and the USA are placing a particular emphasis on increasing the amount and diversity of floral resources within agricultural landscapes without explicitly considering landscape pattern. Just a few strategies consider landscape coplanarity and connectivity. Moreover, most strategies differ in approaches to landscape scale conservation interventions. Thus, in order to preserve pollinator biodiversity existing strategies need to be improved by focusing simultaneously on habitat quality, complementarity and connectivity. Countries that intend to design and introduce pollinator conservation strategies should take best practices from several national strategies 
in order to ensure effective pollinator conservation at a landscape scale.

I want to express my gratitude to Dr. Sarah Greenwood for her great contribution to my research work as a supervisor. I also want to thank Dr. Tonya Lander, Dr. Sharron Ogle, Dr. Robert Thomas, Dr. Ellie Devenish-Nelson and Dr. Graciela Ruth for their assistance and valuable advice on this research.

\section{References}

1. N. Gallai, J.M. Salles, J. Settle and B.E. Vaissiere, Ecological Economics. 68(3), 810-821 (2009)

2. F. Jauker and F. Walters, Oecologia 156(4), 819-823 (2008)

3. K.A. Orford, I.P. Vaughan and J. Memmot, Proceedings of the Royal Society B. 282(1805), 1-6 (2015)

4. R. Rader, et al, PNAS. 113(1), 146-151 (2016)

5. IPBES Thematic assessment on pollinators, pollination and food production (deliverable 3(a)): Individual chapters and their executive summaries. Annex to document IPBES/4/INF/1/Rev.1 p 868 (2016)

6. Promote Pollintors Coalition of the Willing [online]. Promote Pollinators the Coalition of the Willing on Pollinators. Available from: https:// www.promotepollinators.org/coalition-of-thewilling/ [Accessed on 2 of October 2020] (2020)

7. D. Vasiliev and S. Greenwood, The Science of the Total Environment 744, 140880 (2020)

8. D. Senapathi, Current Opinion in Insect Science 12, 93-101 (2015)

9. L.V. Dicks, D.A. Showler and W.J. Sutherland, Bee Conservation: Evidence for the effects of interventions. Exeter: Pelagic Publishing. Synthesis of Conservation Evidence Series p 139 (2010)

10. D.A. Landis, Basic and Applied Ecology. 18, 1-12 (2017)

11. M.G. Betts, A.S. Hadley and U. Kormann, Landscape Ecology. 34, 961-966 (2019)

12. J. Ghazoul, Trends in Ecology and Evolution. 20(7), 367-373 (2005)

13. P. Hoehn, T. Tscharntke, J.M. Tylianakis and I. Steffan-Dewenter, Proceedings of the Royal Society B. 275, 2283-2291 (2008)

14. R.E. Mallinger and C. Gratton, Journal of Applied Ecology. 52, 323-330 (2015)

15. B.R. Blaaw and R. Isaacs, Journal of Applied Ecology. 51(4), 890-898 (2014)

16. M.D. Smith and A.K. Knapp, Ecology Letters. 6, 509-517 (2003)

17. R. Winfree, Science. 359, 791-793 (2018)

18. S. Yachi and M. Loreau, PNAS. 96, 1463-1468 (1999)

19. C. Brittain, Global Change Biology. 19, 540-547 (2013)
20. J. Frund et al. Ecology. 94(9), 2042-2054 (2013)

21. T. Elmquist et al, Frontiers in Ecology and Evolution. 1(9), 488-494 (2013)

22. I. Barthomeus et al, Ecology Letters. 16, 1331-1338 (2013)

23. J.M. Tylanakis, Ecology Letters. 11, 1351-1363 (2008)

24. Winfree R and Kremen C Ecology Letters. 10, 11051113 (2009)

25. T.M. Knight, et al. Ecology Letters. 21(8), 12821295 (2018)

26. D. Goulson, et al. Science. 347(6229), 1435-1445 (2005)

27. J.P. Gozalez-Varo, et al. Trends in Ecology \& Evoultion. 28(9), 524-530 (2013)

28. C. Kremen, et al. PNAS. 99(26), 16812-16816 (2002)

29. T. Blacquière, et al. Ecotoxicology. 21(4), 973-992 (2012)

30. T. Tscharnthe, et al. Biological Conservation. 151(1), 53-59 (2016)

31. Stockstad, E. Science. 340(6133), 674-676 (2013)

32. A.M. Klein, et al. Proceedings of the Royal Society B-Biological Sciences. 274, 303-313 (2007)

33. T. Newbold, et al. PLoS Biology. 16(12), 1-24 (2018)

34. C.M. Kennedy, et al. Ecology Letters. 16(5), 584-599 (2013)

35. N. Deguines, et al. Frontiers in Ecology and the Environment. 12(4), 212-217 (2014)

36. N.M. Williams, et al. Biological Conservation. 143(10), 2280-2291 (2010)

37. P. Fayt, et al. Journal of Insect Conservation. 10, 129-150 (2006)

38. H. Taki, et al. PLoS ONE. 8(2), 1-8 (2013)

39. R.V. Cartar, Biodiversity and Conservation. 14, 1895-1907 (2005)

40. J.L. Hanula et al. Forest Ecology and Management. 348, 142-152 (2015)

41. J. Lindgren, et al. Agriculture, Ecosystems and Environment. 251, 107-113 (2018)

42. Z. Liu, et al. Landscape Ecology. 29(5), 763-771 (2014)

43. A.J. Bates, et al. PLoS ONE. 6(8), 1-11 (2011)

44. A.L. Carper, et al. Environmental Entomology. 43, 253-262 (2014)

45. J.L. Osborne et al. Journal of Applied Ecology. 45, 784-793 (2008)

46. S.J. Hegland, et al. Ecology Letters. 12(2), 184-195 (2009)

47. D. Vasiliev \& S. Greenwood. Science of the Total Environment. 775, 145788 (2021)

48. J.T. Kerr, et al. Science. 349(6244), 177-180 (2015)

49. I.-C. Chen, et al. Science. 333(6045), 1024-1026.

50. J. Settele, BioRisk. 1, 1-712 (2008).

51. P. Rasmont, et al. BioRisk. 10, 1-236 (2015) 
52. B.J. Phillips, et al. Global Change Biology. 24, 32263235 (2017)

53. P. Rasmont and S. Isrbyt. Annales de la Societe Entomologique de France. 48, 275-280 (2012)

54. L. Hannah, et al. Climatic Change. 141, 63-75 (2017)

55. D. Kleijn, et al. Ecology Letters. 9, 243-254 (2006).

56. J. Schepher, et al. Ecology Letters. 16(7), 912-920 (2013)

57. G. Shackelford, et al, Biological Review. 88, 10021021 (2013)

58. D.J. Gonthier, Proceedings: Biological Sciences. 281(1791), 1-8 (2014)

59. R. Winfree et al, Annual Review of Ecology, Evolution, and Systematics. 42, 1-22 (2011)

60. T.H. Roulston and K. Goodell, Annual Reviews of Entomology. 21(8), 293-312 (2011)

61. I. Grass et al, Agriculture, Ecosystems \& Environment. 225, 45-53 (2016)

62. A. Guisan and W. Thuiller, Ecology Letters. 8, 9931009 (2005).

63. D. Senapathy et al, Functional Ecology. 31, 26-37 (2017)

64. J. Pykala, Conservation Biology. 14(3), 705-712 (2000)

65. R.E. Plotnick and R.H. Gardner, Ecological Modelling. 147(2), 171-197 (2002)

66. T.J. Wallington et al, Ecology and Society. 10(1), 116 (2005)

67. J.H. Connell, Science. 199, 1302-1310 (1978)

68. Z.Y. Yuan, Scientific Reports. 6(22132), DOI: 10.1038 (2016)

69. J.R. Hudson, Biological Conservation. 167, 355-362 (2013)

70. H. Taki et al, Forest Ecology and Management. 259, 607-613 (2010)

71. S.H. Black et al, Rangelands. 33(3), pp. 9-13 (2011)

72. M.G. Morris, Biological Conservation. 95, 121-126 (2000)

73. S.D. Fuhlendorf and D.M. Egle BioScience. 51, 625632 (2001)

74. J. Brown et al, Journal of Applied Ecology. 54, 313322 (2017)

75. E. Proctor, et al. Biodiversity Conservation. 21, 1703-1740 (2012)

76. P. Chesson, Theoretical Population Biology. 58, 211-237 (2000)

77. A. Bolin et al, Oikos. 127, 1575-1585 (2018)

78. C. Westphal et al, Oecologia. 149, 289-300 (2006)

79. A.J. Vanbergen, Trends in Ecology and the Environment. 11, 251-259 (2013)
80. T.H. Oliver and M.D. Morecroft, WIRES Climate Change. 5(3), 317-335 (2014)

81. T.H. Oliver et al, Ecology Letters. 13, $473-484$ (2010)

82. L. Fahrig et al. Ecology Letters. 14, 101-112 (2011)

83. J. Bengtsson et al, The Journal of Applied Ecology. 42(2), 261-269 (2005)

84. S.L. Tuck et al, Journal of Applied Ecology. 51, 746755 (2014)

85. G. Carre et al. Agriculture, Ecosystems \& Environment. 133(1-2), 40-47 (2009).

86. C. Schuepp et al, Proceedings of the Royal Society B: Biological Sciences. 281, DOI: 2013.2667 (2014)

87. L.J. Cole et al, Journal of Applied Ecology. DOI: 10.1111/1365-2664.13572 (2020)

88. E. Underwood et al, Pollinator initiatives in EU Member States: Success factors and gaps. Report for European Commission under contract for provision of technical support related to Target 2 of the EU Biodiversity Strategy to 2020 - maintaining and restoring ecosystems and their services ENV.B2/SER/2016/2018. Institute for European Environmental Policy, Brussels (2017)

89. L.G. Caravelherio et al, The Journal of Applied Ecology. 47(4), 810-820 (2010).

90. P. Fayt et al, Journal of Insect Conservation. 10, $129-150$

91. H. Taki et al, PloS One. 8(2), e56678, (2013).

92. R. Fuchs et al, Global Change Biology. 21(1), 299313 (2015).

93. K.C. Ryan et al, Frontiers in Ecology \& the Environment. 11(s1), e15-e24 (2013)

94. FAO, Global Forest Resources Assessment. How are the world's forests changing. Food and Agriculture Organization of the United Nations, Rome (2015).

95. S. Sloan and J.A. Sayer, Forest Ecology and Management. 352, 134-145 (2005).

96. A.R. Nell and K.J. Puettmann, Canadian Journal of Forest Research. 43, 428-440 (2013).

97. W. Proesmans et al, Basic and Applied Ecology. 34, 95-107 (2019).

98. R.V. Cartar, Biodiversity and Conservation. 14, 1895-1907 (2005).

99. J.L. Hanula et al, Forest Ecology and Management. 348, 142-152 (2015).

100.J. Lindgren et al, Agriculture, Ecosystems and Environment. 251, 107-113 (2018).

101.S. Frank et al, Climatic Change. 138(1-2), 253-266 (2016). 